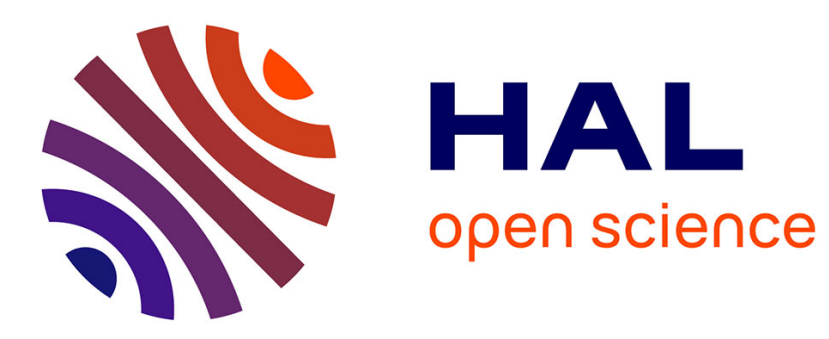

\title{
Collective orientation of an immobile fish school and effect on rheotaxis
}

\author{
Renaud Larrieu, Catherine Quilliet, Aurélie Dupont, Philippe Peyla
}

\section{To cite this version:}

Renaud Larrieu, Catherine Quilliet, Aurélie Dupont, Philippe Peyla. Collective orientation of an immobile fish school and effect on rheotaxis. Physical Review E , 2021, 103 (2), 10.1103/PhysRevE.103.022137 . hal-03149531

\section{HAL Id: hal-03149531 \\ https://hal.science/hal-03149531}

Submitted on 18 Nov 2021

HAL is a multi-disciplinary open access archive for the deposit and dissemination of scientific research documents, whether they are published or not. The documents may come from teaching and research institutions in France or abroad, or from public or private research centers.
L'archive ouverte pluridisciplinaire HAL, est destinée au dépôt et à la diffusion de documents scientifiques de niveau recherche, publiés ou non, émanant des établissements d'enseignement et de recherche français ou étrangers, des laboratoires publics ou privés. 


\title{
Collective orientation of an immobile fish school and effect on rheotaxis
}

\author{
Renaud Larrieu $\odot$, Catherine Quilliet, Aurélie Dupont, and Philippe Peyla ${ }^{*}$ \\ Université Grenoble Alpes, Centre National de la Recherche Scientifique, LIPhy, F-38000 Grenoble, France
}

(Received 4 November 2020; accepted 5 February 2021; published 22 February 2021)

\begin{abstract}
We study the orientational order of an immobile fish school. Starting from the second Newton law, we show that the inertial dynamics of orientations is ruled by an Ornstein-Uhlenbeck process. This process describes the dynamics of alignment between neighboring fish in a shoal—a dynamics already used in the literature for mobile fish schools. First, in a fluid at rest, we calculate the global polarization (i.e., the mean orientation of the fish), which decreases rapidly as a function of noise. We show that the faster a fish is able to reorient itself the more the school can afford to reorder itself for important noise values. Second, in the presence of a stream, each fish tends to orient itself and swims against the flow: so-called rheotaxis. So, even in the presence of a flow, it results in an immobile fish school. By adding an individual rheotaxis effect to alignment interaction between fish, we show that in a noisy environment individual rheotaxis is enhanced by alignment interactions between fish.
\end{abstract}

DOI: 10.1103/PhysRevE.103.022137

\section{INTRODUCTION}

The appearance of self-organization within a group of active entities is a fascinating phenomenon [1]. It has been studied for micro-organisms $[2,3]$ as well as for active synthetic particles [4,5] and at larger scales for animals [6]. Nontrivial self-organization is also observed within fish shoals [7-10]. Milling and schooling are collective phenomena occurring on scales much larger than an individual fish. This phenomenon has been studied since the beginning of the 20th century [11]. The structure and the sensitivity to external factors such as water temperature, light, and darkness were analyzed by Breder [12]. Individual fish in a school were observed to swim for a longer duration when aligned, with lower tail-beat frequencies and smaller energy dissipation and respiratory rates, compared to fish swimming alone [13-15]. In addition, shoaling and alignment between fish are established as a result of many social and sensory factors like metabolism [16] and alignment by vision [17] or food [18]. Recently, the study of out-of-equilibrium active systems [19] allowed scientists to substantially improve their knowledge in modeling this remarkable phenomenon. In the seminal work of Vicsek et al. [20], an individual animal (bird or fish) adopts instantaneously the average orientation of its neighbors in the group, resulting in a collective motion that can be destroyed by noise. The noise source can be intrinsic to the fish or due to external conditions such as turbulent fluid flow [9]. Since then, more sophisticated force models have emerged that reproduce quite well the real behavior of schools of fish [21-23]. That class of social model allows one to study several situations with some flexibility [24].

If collective motions have been extensively studied [1], quite little literature is devoted to immobile groups of fish [25] which stay at the same place relative to their living en-

\footnotetext{
*philippe.peyla@univ-grenoble-alpes.fr
}

vironment. Immobile fish schools can be observed in various situations and especially in reef regions [26]. Origins of such an immobile state are diverse. It is likely that schools of fish that stop their movements and remain motionless for a period of time may achieve perceptual benefits [10]. Simultaneous stopping of a school of fish provides relatively quiet intervals to allow reception of potentially critical environmental signals; fish under predator threat that form nonmoving look around shoals [7] may be an example. However, the most frequent origin of immobile school is a rheotactic effect that allows the fish to orient against a stream [8] and is the object of the present model. This effect was studied in detail by Potts [27]. A school of the snapper Lutjanus monostigma was observed during several days and self-organized into a polarized and immobile school when submitted to tidal flow. Each fish was heading towards the current in order to maintain its position by positive rheotaxis. This is done by swimming gently at an equal and opposite speed to the current. Indeed, pointing ahead in a direction opposed to the flow can help the school to maintain its immobility in a region where food is present. A fish can individually find the direction of flow through sensitive captors $[28,29]$ and can also try to align with its congeners. Note that within our simple model the notion of rheotaxis can be extended to taxis in general: each fish tends to orient itself in a particular direction while maintaining a fixed position in space.

In the following, we will first present the model of fish orientation with respect to neighbors and flow. We then show that alignment interactions within a shoal can increase rheotaxis efficiency of a single fish.

\section{MODEL}

Let us consider a motionless fish (Fig. 1) located at a fixed position and with a time-varying orientation, living in a school and interacting with its neighbors while attempting to orient itself in a direction opposite to a uniform flow (rheotaxis). Here, 


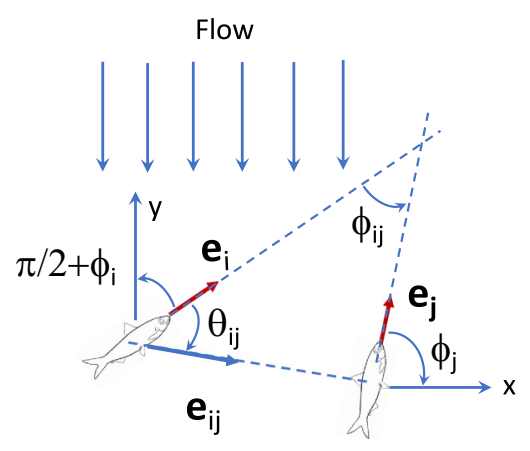

FIG. 1. Fish in a flow. The flow is oriented toward the $y<0$ direction. Angles: $\phi_{i}$ is the angle between orientation $\mathbf{e}_{i}$ of the $i$ th fish and the horizontal $x$ axis; $\theta_{i j}$ is the angle between fish $i$ orientation $\mathbf{e}_{i}$ and fish $i$ - $j$ orientation vector $\mathbf{e}_{i j}$; finally $\phi_{i j}$ is the angle between orientations of fish $i$ and $j$.

for simplicity we use a two-dimensional fish shoal [22,23] with a circular shape of radius $R$. Each of the $N$ fish is a discoid of radius $a$. When we vary $N$, we maintain the density $\rho=N(a / R)^{2}$ constant, typically, $\rho \approx 0.5$. This corresponds to a quite dense school which can be often encountered [30] and which allows us to have small fluctuations of the local density of fish. In the spirit of the social force model originally developed by Helbing et al. [31,32], we consider here social torques. These "torques" are a measure for the internal motivations of each individual to perform certain movements (rotation) depending on its environment. Each fish needs to orient itself in the same direction as its neighbors within a chosen radius of $5 a$ (i.e., 2.5 times its own size) and against the flow. The typical size $5 a$ has been chosen to capture neighbors that are closely neighboring a given fish regarding the chosen density $\rho \approx 0.5$, and represents on average 12.5 fish. For $N=200$ fish, the total school size is $R=20 a$, which is four times bigger than the radius of interaction. Starting with the second Newton law for rotating bodies we can write

$$
\mathcal{I} \dot{\omega}_{i}=-\zeta \omega_{i}+\sum_{j \in V_{i}} T_{i j}^{I}\left(\phi_{i j}\right)+T_{i}^{R}\left(\phi_{i}\right)+\eta\left(\mathbf{r}_{i}, t\right),
$$

where $\mathcal{I}$ is the moment of inertia of any fish (supposed identical), and $\omega_{i}$ is the angular velocity of fish $i$ located in $\mathbf{r}_{i}$ and oriented with an angle $\phi_{i}$ with the $x$ axis at time $t$. The angular acceleration is $\dot{\omega}_{i}$. Fluid friction is $\zeta$. The $i$ th fish interacts with its close congeners indexed by $j$ in the $V_{i}$ ensemble of its $N_{i}$ neighbors (i.e., within a circle of radius $5 a$ around fish $i$ ). This interaction is represented by a social torque $T_{i j}^{I}$ which depends on the relative orientation between fish $i$ and $j: \phi_{i j}=\phi_{i}-\phi_{j}$. The torque associated with the rheotaxis is $T_{i}^{R}$, which depends on the orientation of the fish $i$ with the $y$ axis chosen as the direction of the incident and uniform flow. Finally, the dynamics is perturbed by a noise term $\eta(\mathbf{r}, t)$ with $<\eta(\mathbf{r}, t)>=0$ and $<\eta(\mathbf{r}, t) \eta\left(\mathbf{r}^{\prime}, t^{\prime}\right)>\sim \delta\left(\mathbf{r}-\mathbf{r}^{\prime}\right) \delta\left(t-t^{\prime}\right)$. Note that, in our model, we do not consider an interaction term that depends explicitly on fish interdistance. This equation can be rewritten as

$$
\dot{\omega}_{i}=-\frac{1}{\tau} \omega_{i}+\frac{1}{\tau} \omega_{i}^{*}\left(\phi_{i j}\right)+\eta\left(\mathbf{r}_{i}, t\right) / \mathcal{I},
$$

which expresses that each fish adjusts its angular velocity $\omega(t)$ towards a time-dependent target value $\omega_{i}^{*}=\omega_{i}^{I *}+\omega_{i}^{R *}$, depending both on the fish-fish interaction $\left(\omega_{i}^{I *}\right)$ and on the rheotaxis $\left(\omega_{i}^{R *}\right)$ within an external flow. Expressions of $\omega_{i}^{I *}$ and $\omega_{i}^{R *}$ are given below. We use the time $\tau$ associated with dissipation as the characteristic time and we rescale by $\tau$ the other times associated with alignments and rheotaxis. Let us consider a fish as a rigid and prolate ellipsoid with a size around a few centimeters at a fixed position in a flow with a velocity $U_{0}$ at intermediate Reynolds numbers $(\approx 100)$. The torque exerted by the fluid on the ellipsoid is $T \approx 1 / 2 \rho U_{0}^{2}\left(\pi / 8 d^{3}\right)$ [33] where $d$ is the diameter of the equivalent sphere and $\rho$ is the water density. The momentum of inertia of a prolate ellipsoid rotating around its minor axis is $\mathcal{I}=m\left(b^{2}+c^{2}\right) / 5$ where $b$ and $c$ are the semiminor and semimajor axis, respectively, and $m$ is the mass of the fish supposed to be neutrally buoyant, i.e., $m=4 / 3 \rho \pi b^{2} c=$ $1 / 6 \rho \pi d^{3}$. Thus, the angular acceleration is $T / \mathcal{I}$. If we assume that the fish rotate at an angle $\pi / 2$ on a time scale $\tau$, it gives $\tau^{2}=(\pi / 2) \mathcal{I} / T$. This leads to a time $\tau$ of about a few seconds, which is much larger than the typical time of reaction for alignment (of the order of a few tenths of a second [21]). However, the time associated with dissipation can be much shorter if we consider that usually a fish is flexible and a change of orientation is driven by a deformation of its body which can greatly reduce its inertia [34]. Following the spirit of $[22,23]$ in order to describe the interaction between close fish, we write

$$
\omega_{i}^{I *}=\frac{I}{\tau} \frac{1}{N_{i}} \sum_{j \in V_{i}} \sin \left(\frac{\phi_{i j}}{2}\right) \frac{1+\cos \left(\theta_{i j}\right)}{2},
$$

where $I$ is the dimensionless amplitude of alignment interaction. The term $\sin \left(\phi_{i j} / 2\right)$ accounts for alignment between fish $i$ and $j$. If fish $i$ and $j$ are aligned in the same direction, it reads $\sin \left(\phi_{i j} / 2\right)=0$ and then $\omega_{i}^{*}=0$ since fish $i$ has no reason to rotate. But if fish $i$ and $j$ are antiparallel, i.e., $\phi_{i j}= \pm \pi$, then $\sin \left(\phi_{i j} / 2\right)= \pm 1$, since fish $i$ must rotate. The term $\left(1+\cos \theta_{i j}\right) / 2$ is designed to ensure a frontal preference and some kind of rear blind angle [23]. In order to model the alignment against the flow (rheotaxis), we have

$$
\omega_{i}^{R *}=\frac{F}{\tau} \sin \left(\frac{\pi / 2+\phi_{i}}{2}\right) .
$$

The term $F$ is dimensionless and represents the amplitude of the rheotaxis. If $\phi_{i}=-\pi / 2$, the fish does not rotate since it is already aligned against the flow (i.e., pointing in the $y>0$ direction), which reads $\omega_{i}^{R *}=0$. But if the fish is aligned along the flow, $\left(\phi_{i}=+\pi / 2\right)$, the fish must turn back in order to point against the flow with the target angular velocity $\omega_{i}^{R *}=F / \tau$.

Using an Euler-Maruyama integration [35], Eq. (2) reads

$$
d \omega_{i}(t)=-\frac{d t}{\tau}\left[\omega_{i}(t)-\omega_{i}^{*}(t)\right]+\sigma \mathcal{N} \sqrt{d t},
$$

also known as an Ornstein-Uhlenbeck process [21,22]. The noise amplitude is $\sigma$ and $\mathcal{N}$ is a random Gaussian variable of 
mean zero and variance 1 . For large values of time, this equation becomes stationary (see the Appendix). In the absence of rheotaxis $(F=0)$, we can rescale time and angular velocities by $\tau / I$ and $I / \tau$, respectively. At stationarity, it is easy to show that there is only one dimensionless number $\tilde{\sigma}=\sigma \tau^{3 / 2} / I^{1 / 2}$ that compares the amplitude of noise and the amplitude of alignment interaction (see the Appendix).

In the presence of rheotaxis $(F \neq 0)$, three terms should be compared: the alignment interaction (amplitude $I$ ), the rheotaxis (amplitude $F$ ), and the noise term (amplitude $\sigma$ ). Since we would like to vary $F$ at constant $I$, we choose to rescale time and angular velocities by $\tau / F$ and $F / \tau$, respectively. Then, we get two dimensionless numbers $I / F$ and $F^{1 / 2} /\left(\sigma \tau^{3 / 2}\right)$, the last term being equal to $(F / I)^{1 / 2} / \tilde{\sigma}$.

In the following, we will integrate numerically Eq. (5) in the absence or in the presence of rheotaxis. For each situation, we will plot the global polarization (defined below) as a function of the above dimensionless numbers.

\section{RESULTS}

We first analyze the alignment within an immobile school of $N=200$ fish as a function of the noise $\tilde{\sigma}$ and without rheotaxis $(F=0)$. Note that we tried different numbers of fish: $N=400,200,100$, and 50 (see the Appendix). Since results are quite close for $N=400$ and 200, we choose to work with $N=200$ fish for this paper.

We compute the mean value of the global polarization [20,22] defined as $P=\frac{1}{N}\left|\sum_{i=1}^{N} \mathbf{e}_{i}\right|$ :

$$
P=\frac{1}{N} \sqrt{\left(\sum_{i=1}^{N} \cos \phi_{i}(t)\right)^{2}+\left(\sum_{i=1}^{N} \sin \phi_{i}(t)\right)^{2}} .
$$

To calculate $P$, we run 20 simulations for each set of parameters. Each simulation is done with a different configuration of fish. This number of runs seems sufficient to reduce the fluctuations of $P$ due to the choice of configuration. The global polarization of the school is $P=1$ when all fish point in the same direction while $P=0$ means that the fish point in different directions.

The school initial orientation is polarized in a given random direction. After a transient time of order $\tau / I$, fish can lose partially their mutual alignment because of the noise. Thus, we measure $P$ for large values of time $(t \gg \tau / I)$ as a function of $\tilde{\sigma}$ (see Fig. 2). We found that $P$ drops abruptly for $\tilde{\sigma} \sim 0.27$. Here, the rescaled noise being $\tilde{\sigma}=\sigma \tau^{3 / 2} / \sqrt{I}$, which means that fast reacting fish (small values of $\tau / I$ ) are more able to line up in a more noisy environment (large value of $\sigma$ ) than fish with a larger $\tau / I$ value that cannot sustain the same amplitude of noise to form a polarized school. Note also that when $P=0.6 \pm 1$ we obtain a maximum of polarization fluctuations whatever $N$ (see the Appendix, Fig. 8). This is reminiscent of phase transitions where fluctuations of a finite system are maximum at the transition.

To visualize the loss of orientation when noise increases, let us first consider the map of individual polarizations around $\tilde{\sigma}=0.27$. In Fig. 3, we plot the time averaged value of each direction $\overline{\mathbf{e}}_{i}(t)=1 / t_{\max } \int_{0}^{t \max } \mathbf{e}_{i} d t$ for different values of $\tilde{\sigma}$. We use $t_{\max } /(\tau / I)=10^{4}$ and $d t /(\tau / I)=10^{-2}$. Starting with

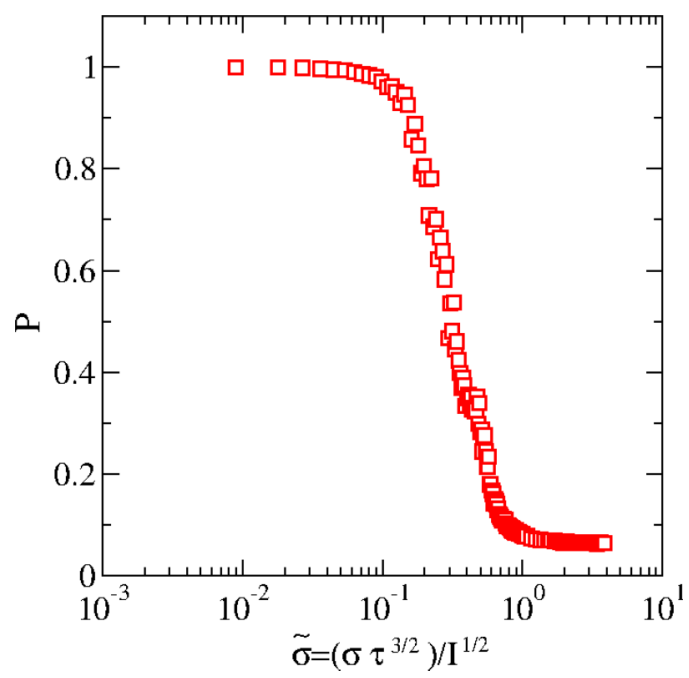

FIG. 2. Decrease of the global polarization for $N=200$ fish as a function of the dimensionless noise $\tilde{\sigma}$ without rheotaxis $(F=0)$. Each point is averaged over 20 runs. The total time of each simulation is $t_{\max } /(\tau / I)=10^{4}$ with a time step $d t /(\tau / I)=10^{-2}$.

$\tilde{\sigma}=0.18$, the group of fish is well polarized [Fig. 3(a)]. Then, we increase the rescaled noise value. We see that for $\tilde{\sigma}=0.27$ [Fig. 3(b)] a weak polarized region appears. Around $\tilde{\sigma}=0.54$ [Fig. 3(c)] several weak polarized zones have invaded the school, leading to islands of polarized fish separated by unpolarized zones. For larger values $\tilde{\sigma}=1.0$ [Fig. 3(d)], an entire unpolarized fish school (a so-called shoal) remains. We also calculate the correlation function $C=$ $\overline{\mathbf{e}_{i} \cdot \mathbf{e}_{j}}=1 / t_{\max } \int_{0}^{t \max } \mathbf{e}_{i} \cdot \mathbf{e}_{j} d t$ for different distances $d$ between fish $i$ and $j$. As shown in Fig. 4, $C$ decreases exponentially as a function of the fish-to-fish distance with a typical correlation length $\lambda$. This correlation length decreases by increasing the noise (Fig. 5). We observe a small plateau for $\lambda$ close to the school size $R$ probably due to a boundary effect.

To study the effect of the alignment interaction between fish on the rheotaxis of the whole group, we now consider nonzero values of $F$. In the absence of alignment interactions between individuals $(I=0)$, each fish tends to orient itself against the flow (pointing toward the $y>0$ direction). The presence of noise perturbs the rheotactic behavior of each fish and the polarization drops by increasing the noise $\sigma$ or $\tau$ and decreasing the rheotaxis $F$ (Fig. 6). We assume that even if a given fish is not perfectly oriented against the flow it still maintains its position within the shoal in order to stay with its congeners.

Now, by switching on the alignment interaction between fish (i.e., for nonzero and positive values of $I$ ), we observe a clear increase of the global polarization [see Fig. 6 (black arrow)]. Note that the $x$ axis $\left[\sqrt{F} /\left(\sigma \tau^{3 / 2}\right)\right]$ is inversely proportional to $\sigma$. In Fig. 6(b), we plot the polarization difference $\Delta P=P(I / F)-P(I / F=0)$ between the global polarization $P$ of the school in the presence of interactions and in the absence of social interaction $(I=0)$. We see that for large values of $I / F$ a strong increase of the polarization against the flow is obtained and reaches a maximum around $F^{1 / 2} /\left(\sigma \tau^{3 / 2}\right) \approx 1$. For small rheotaxis or strong noise the global polarization drops to zero since the fish are pointing in all directions. In 

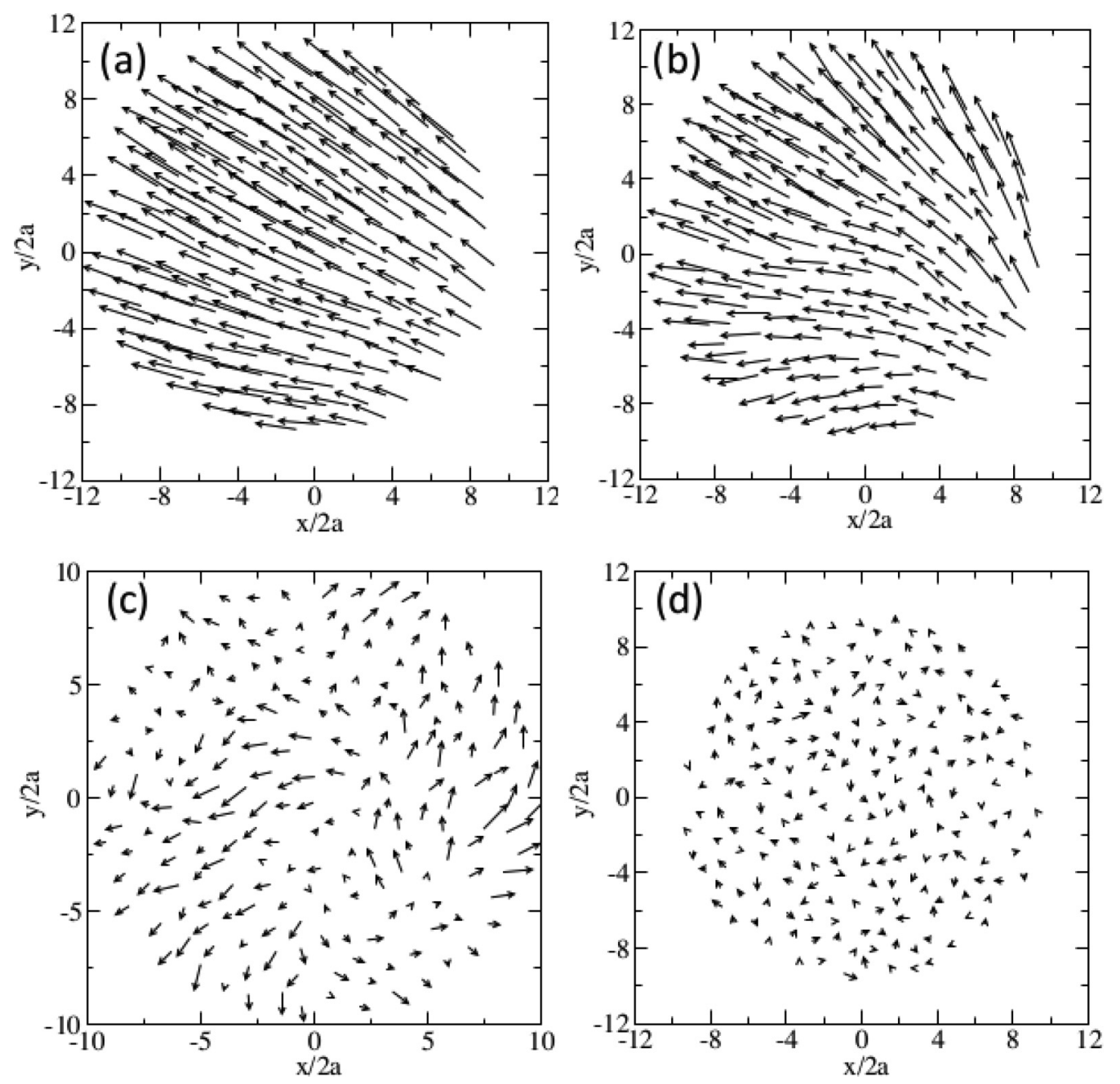

FIG. 3. Time averaged orientations of each fish for different values of the noise without rheotaxis $(F=0)$. A short or large arrow indicates small or large polarization of a given fish, respectively. (a) For $\tilde{\sigma}=0.18$, fish are well polarized. (b) For $\tilde{\sigma}=0.27$, a small zone of weak polarization appears (bottom right). (c) For a larger value $\tilde{\sigma}=0.54$, only a few islands of polarized fish remain. (d) Then, for $\tilde{\sigma}=1.0$, no polarization remains (time averaged value $\overline{\mathbf{e}}_{i} \approx 0$ ).

contrast, for large rheotaxis or small noise all the fish are pointing in the direction opposed to the flow and $P$ saturates. In both cases, the role of fish-fish interaction is inefficient. But between these two extreme cases $\left[F^{1 / 2} /\left(\sigma \tau^{3 / 2}\right) \approx 1\right]$ we observe a maximum of $\Delta P$ corresponding to a significant gain of rheotaxis by the interplay of the fish-to-fish interactions. It can be concluded that a strong fish-to-fish interaction improves the collective rheotaxis even if the individual rheotaxis is weak.

\section{CONCLUSION}

In this paper, we have studied the collective orientation of an immobile group of fish with two ingredients: a social torque to align fish with their close neighbors and an environmental torque to align fish with an external flow. We have modeled the inertial dynamics of groups of fish in the presence of noise. In the absence of an external flow, we show that for large values of a dimensionless noise $\tilde{\sigma} \gg 1$ the group cannot globally polarize. In the presence of a flow, we show that strong social interactions help the group to detect and align even with weak individual rheotaxis. We believe that this model, despite its simplicity, could be extended to describe important features regarding the behavior of groups of animals and in particular predator-prey systems. Indeed, pack interactions can improve hunting efficiency for predators or improve the protection of a group of prey.

Beyond the scope of this paper, our model can be extended in many ways to take into account more specific characteristic properties depending on fish species. First, a distance dependent alignment interaction could be included. We can consider that a fish alignment smoothly decreases with the interdistance between individuals (an exponential decay, for example). This decay should depend on density. Indeed, if the density is small, visual interaction range can be large, while at high density the range is very much reduced (as in our model). Second, the ratio between the size of the shoal and the interaction range could also be increased since information propagation delays have been numerically observed in large mobile schools as well as the disappearance of milling configurations [23]. Third, a three-dimensional study would also be very interesting to see the effect of an additional degree 


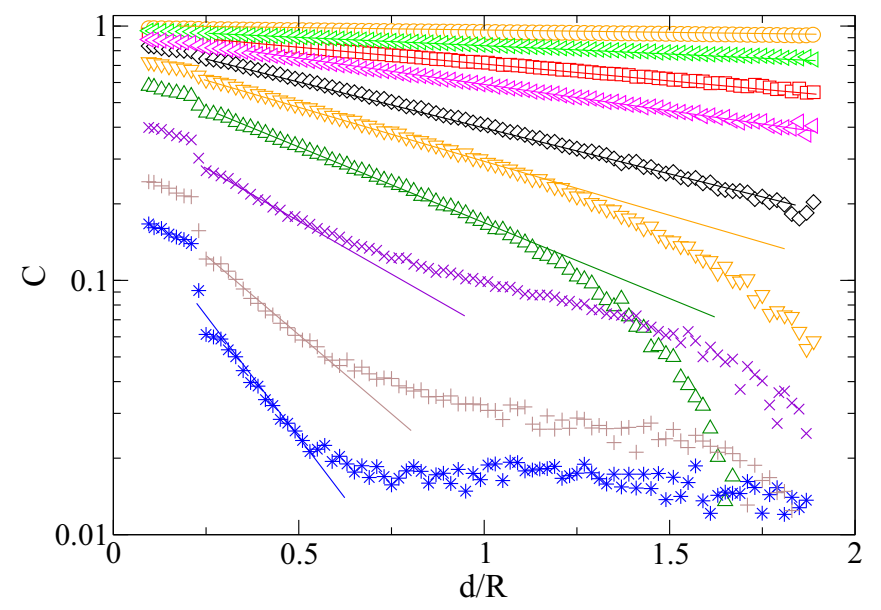

FIG. 4. Correlation function $C$ as a function of the fish-to-fish distance $d$ rescaled by $R$. The first part of the curves is associated with the correlation between close neighbor fish $(d<5 a)$. Then, for $d>5 a$, an exponential decay is observed. Orange circles, $\tilde{\sigma}=$ 0.09; green left triangles, $\tilde{\sigma}=0.13$; red squares, $\tilde{\sigma}=0.18$; magenta down triangles, $\tilde{\sigma}=0.22$; black diamonds, $\tilde{\sigma}=0.27$; orange down triangles, $\tilde{\sigma}=0.36$; green up triangles, $\tilde{\sigma}=0.45$; purple crosses, $\tilde{\sigma}=0.54$; brown plus, $\tilde{\sigma}=0.63$; blue stars, $\tilde{\sigma}=0.72$. Each solid line is a fit with an exponential decay such as $\exp (-d / \lambda)$, where $\lambda$ is a correlation length (fitting parameter).

of freedom on both the polarization and the coupling between fish-fish interaction and rheotaxis.

Our model could also be extended to the case of moving fish like larval zebrafish, which are able to detect low gradients of velocities (in a Poiseuille flow) [36]. In this case, it would be interesting to study whether collective social interactions can improve the efficiency of this specific rheotactic behavior.

All those possible extensions show that our model is quite plastic and can be easily adapted to several situations and can capture several rheotaxis dynamics of a school in a complex environment.

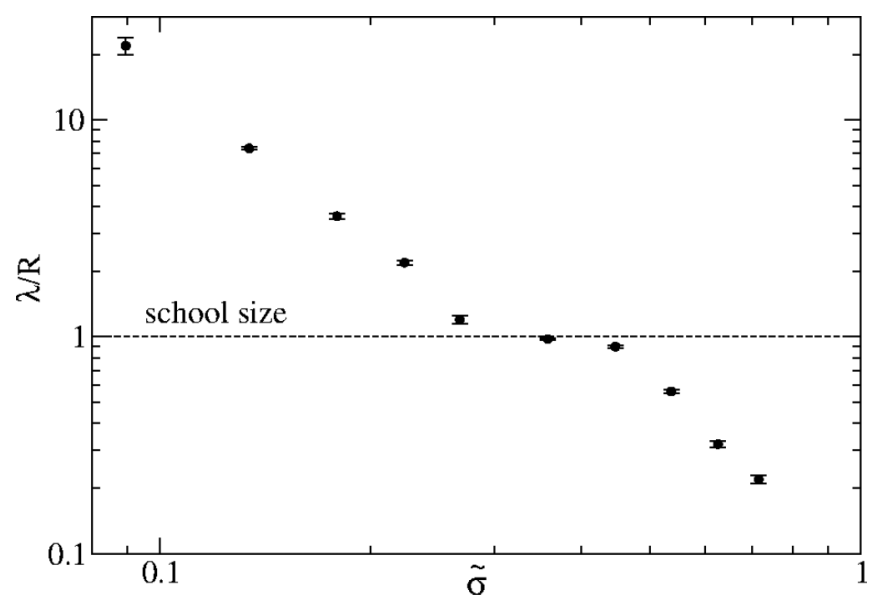

FIG. 5. Correlation length $\lambda$ as a function of noise $\tilde{\sigma} . \lambda$ decreases as a function of $\tilde{\sigma}$ and reaches a plateau when becoming comparable to the school size $R$ around $\tilde{\sigma} \approx 0.27$. Above this noise it continues to decrease below $R$.
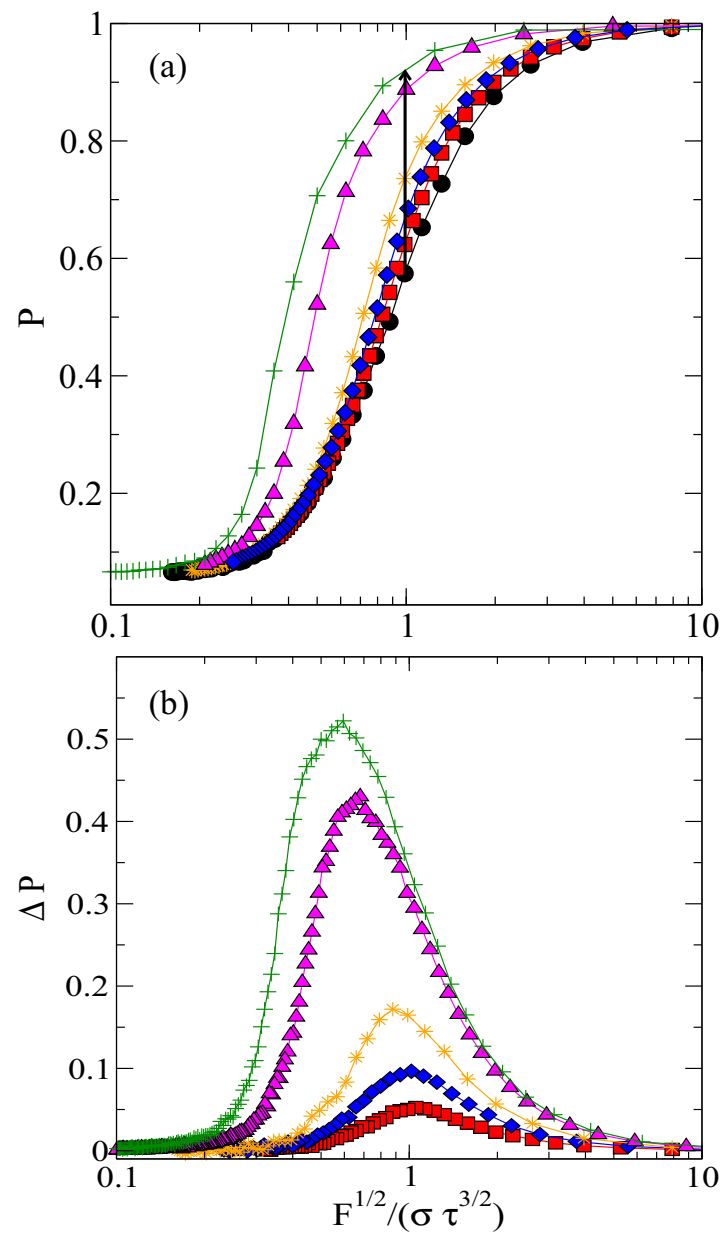

FIG. 6. (a) Polarization $P$ of the school against the flow as a function of $F^{1 / 2} /\left(\sigma \tau^{3 / 2}\right)$ for different values of the parameter $I / F . I / F=$ 20.0 (green crosses), $I / F=10.0$ (magenta triangles), $I / F=2.0$ (orange stars), $I / F=1.0$ (blue diamond), $I / F=0.5$ (red squares), $I / F=0$ (black circles). A significant increase of $P$ is observed when $I / F$ increases. The arrow indicates the polarization difference between $I / F=20.0$ and 0 at $F^{1 / 2} /\left(\sigma \tau^{3 / 2}\right)=1$. (b) Polarization difference of the school $\Delta P=P(I / F)-P(I / F=0)$. The colors and symbols represent the same $I / F$ values as in figure (a). The presence of alignment interaction is very efficient for rheotaxis when $I / F$ is large and $F^{1 / 2} /\left(\sigma \tau^{3 / 2}\right) \approx 1$.

\section{ACKNOWLEDGMENTS}

This project has received financial support from the Centre National de la Recherche Scientifique through the Mission pour les Initiatives Transverses et Interdisciplinaires interdisciplinary programs. We thank our colleagues P. Moreau and P. Ballet for their technical help.

\section{APPENDIX}

\section{Different numbers of fish}

We vary the number of fish, from $N=50$ to 400 (see Fig. 7). Depending on $N$, the drop of polarization occurs in the range of noise $0.25<\tilde{\sigma}<0.5$, however the amplitude of fluctuation always occurs around $P=0.6 \pm 0.1$ (Fig. 8). By changing $N$, we maintain the density $\rho=N /\left(\pi R^{2}\right)$ constant and equal to $\rho \approx 0.5$. 


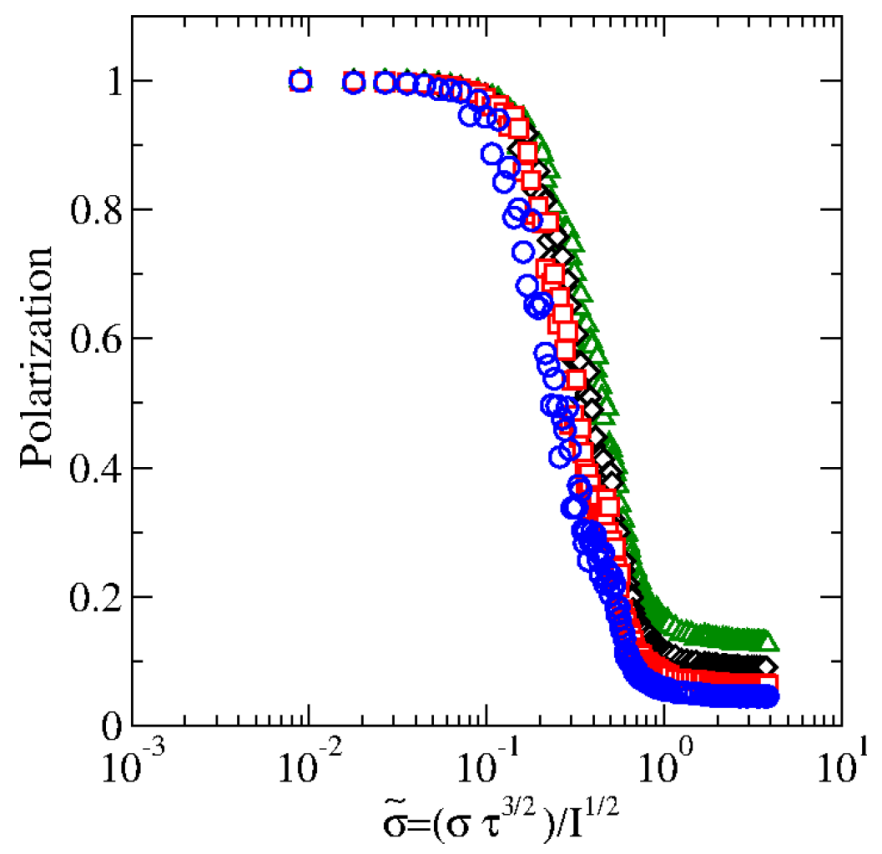

FIG. 7. Polarization of the school as a function of noise $\tilde{\sigma}$ for different numbers of fish $N$. Green triangles, $N=50$; black diamonds, $N=100 ;$ red squares, $N=200$; blue circles, $N=400$.

\section{Dimensionless numbers}

In the absence of rheotaxis $(F=0)$ the rescaling of Eq. (5) at stationarity (see Sec. II) leads to

$$
0=-\tilde{d} t\left[\tilde{\omega}_{i}(t)-\tilde{\omega}_{i}^{*}(t)\right]+\frac{\sigma \tau^{3 / 2}}{I^{1 / 2}} \mathcal{N} \sqrt{\tilde{d} t}
$$

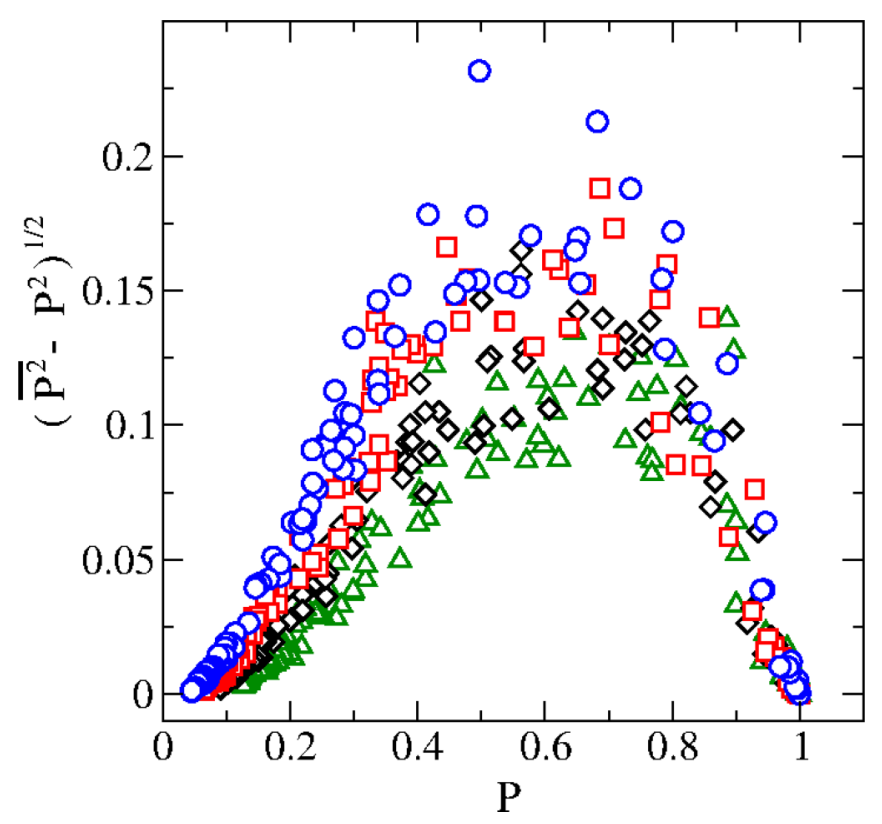

FIG. 8. Fluctuation of the school polarization as a function of polarization for different numbers of fish $N$. Green triangles, $N=50$; black diamonds, $N=100$; red squares, $N=200$; blue circles, $N=$ 400. Whatever $N$, the fluctuation maximum is around $P=0.6 \pm 0.1$.

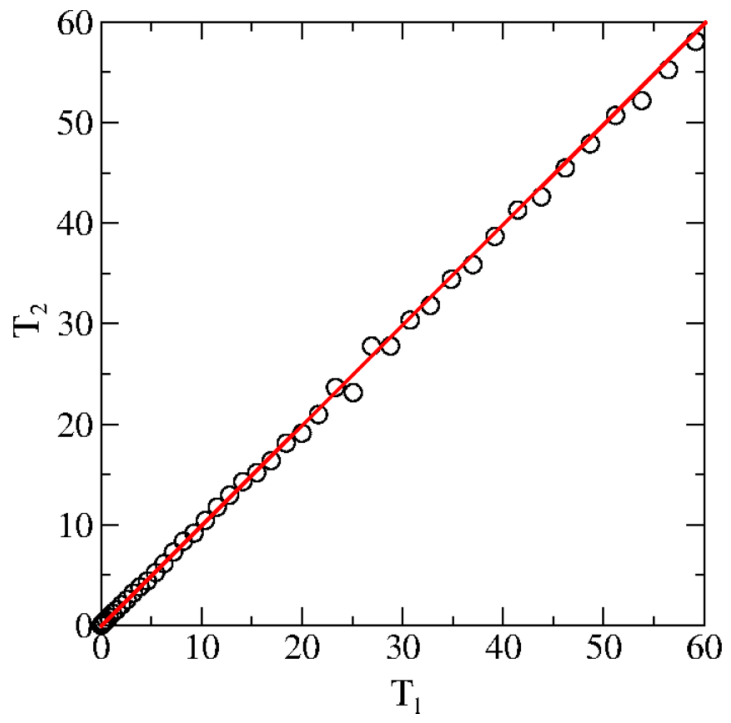

FIG. 9. Term $T_{1}$ [left-hand side of Eq. (A6)] as a function of $T_{2}$ [right-hand side of Eq. (A6)]. Data are circles and the red solid line represents the first bisector, showing that $T_{1}=T_{2}$.

where $\tilde{t}=t I / \tau$ and $\tilde{\omega}=\omega \tau / I$. The dimensionless target is

$$
\tilde{\omega}_{i}^{*}=\frac{1}{N_{i}} \sum_{j \in V} \sin \left(\frac{\phi_{i j}}{2}\right) \frac{1+\cos \left(\theta_{i j}\right)}{2} .
$$

Now, in the presence of rheotaxis $(F \neq 0)$ the rescaling of Eq. (5) at stationarity leads to

$$
0=-\tilde{d} t\left[\tilde{\omega}_{i}(t)-\tilde{\omega}_{i}^{*}(t)\right]+\frac{\sigma \tau^{3 / 2}}{F^{1 / 2}} \mathcal{N} \sqrt{\tilde{d} t}
$$

where $\tilde{t}=t F / \tau$ and $\tilde{\omega}=\omega \tau / F$. The target is

$$
\tilde{\omega}_{i}^{*}=\frac{I}{F} \frac{1}{N_{i}} \sum_{j \in V} \sin \left(\frac{\phi_{i j}}{2}\right) \frac{1+\cos \left(\theta_{i j}\right)}{2}-\sin \left(\frac{\pi / 2+\phi_{i}}{2}\right),
$$

In this case, two dimensionless numbers are to be considered: $I / F$ and $\sqrt{F} /\left(\sigma \tau^{3 / 2}\right)$.

\section{Stationarity}

For large values of time $(\tilde{t} \gg 1)$, we can assume stationarity. In the presence of rheotaxis, if this hypothesis is true, we should have

$$
\left[\tilde{\omega}_{i}-\tilde{\omega}_{i}^{*}\right] \tilde{d} t=\frac{\sigma \tau^{3 / 2}}{F^{1 / 2}} \mathcal{N} \sqrt{\tilde{d} t}
$$

But this equality is not easy to prove numerically because of the presence of noise. So let us average each member of Eq. (A5) on time and fish. By integrating on time and using Ito isometry we obtain the following equality:

$$
\left\langle\left\{\frac{1}{\tilde{t}_{\max }} \int_{0}^{\tilde{t}_{\max }}\left[\tilde{\omega}-\tilde{\omega}^{*}\right] \tilde{d} t\right\}^{2}\right\rangle=\left(\frac{\sigma \tau^{3 / 2}}{F^{1 / 2}}\right)^{2}
$$


where $\tilde{t}_{\max }=t_{\max } /(\tau / F)=10^{4}$ and $<.>$ represents the averaging over the $N$ fish. Let us set $T_{1}=\left\langle\left\{\frac{1}{\tilde{t}_{\max }}\right.\right.$ $\left.\left.\int_{0}^{\tilde{t}_{\max }}\left[\tilde{\omega}-\tilde{\omega}^{*}\right] \tilde{d} t\right\}^{2}\right\rangle$ and $T_{2}=\left(\frac{\sigma \tau^{3 / 2}}{F^{1 / 2}}\right)^{2}$. In Fig. 9, we plot
$T_{1}$ as a function of $T_{2}$ for different values of $\sigma, \tau, I$, and $F$. We show that these two terms are identical and thus the stationarity hypothesis is true when $\tilde{t}_{\max } \gg 1$.
[1] T. Vicsek and A. Zafeiris, Phys. Rep. 517, 71 (2012).

[2] E. B. Jacob, I. Cohen, and H. Levine, Adv. Phys. 49, 395 (2000).

[3] B. Vincenti, G. Ramos, M.-L. Cordero, C. Douarche, R. Soto, and E. Clement, Nat. Commun. 10, 5082 (2019).

[4] W. Paxton, A. Sen, and T. Mallouk, Eur. J. Chem. 11, 6462 (2005).

[5] V. Lobaskin and M. Romenskyy, Phys. Rev. E 87, 052135 (2013).

[6] S. Gueron and S. A. Levin, J. Theor. Biol. 165, 541 (1993).

[7] D. V. Radakov, Schooling in the Ecology of Fish (Wiley, New York, 1973).

[8] D. S. Pavlov and A. O. Kasumyan, J. Ichthyology 40, S163 (2000).

[9] J. Liao, Phil. Trans. R. Soc. B 362, 1973 (2007).

[10] M. Larsson, Curr. Zool. 58, 116 (2012).

[11] A. E. Parr, Bull. Bingham Oceanogr. Collection Yale Univ. 1, 1 (1927).

[12] C. M. Breder, Bull. Am. Mus. Nat. Hist. 98, 1 (1951).

[13] J. C. Svendsen, M. Bildsoe, and J. Steffensen, J. Fish Biol. 62, 834 (2003).

[14] R. M. Ross and T. W. H. Backman, American Shad. Trans. Am. Fish. Soc. 121, 385 (1992).

[15] I. Ashraf, H. Bradshaw, T.-T. Ha, J. Halloy, R. Godoy-Diana, and B. Thiria, Proc. Natl. Acad. Sci. USA 114, 9599 (2017).

[16] F. R. J. Parker, Trans. Am. Fish. Soc. 102, 125 (1973).

[17] B. L. Partridge and T. Pitcher, J. Comp. Physiol. A 135, 315 (1980).

[18] J. Krause, Oecologia 93, 356 (1993).

[19] C. Reichhardt and C. Reichhardt, Soft Matter 14, 490 (2018).

[20] T. Vicsek, A. Czirok, E. Ben-Jacob, I. Cohen, and O. Shochet, Phys. Rev. Lett. 75, 1226 (1995).
[21] J. Gautrais, C. Jost, M. Soria, A. Campo, S. Motsch, R. Fournier, S. Blanco, and G. Theraulaz, J. Math. Biol. 58, 429 (2009).

[22] J. Gautrais, F. Ginelli, R. Fournier, S. Blanco, M. Soria, H. Chaté, and G. Theraulaz, PLoS Comput. Biol. 8, e1002678 (2012).

[23] D. S. Calovi, U. Lopez, S. Ngo, C. Sire, H. Chaté, and G. Theraulaz, New J. Phys. 16, 015026 (2014).

[24] D. S.Calovi, A. Litchinko, V. Lecheval, U. Lopez, A. P. Escudero, H. Chaté, C. Sire, and G. Theraulaz, PLoS Comput. Biol. 14, e1005933 (2018).

[25] W. D. Hamilton, J. Theor. Biol. 31, 295 (1971).

[26] M. H. A. Keenleyside, Diversity and Adaptation in Fish Behaviour, Vol. 11 (Springer, New York, 1979), p. 170.

[27] G. W. Potts, J. Zool. 161, 223 (1970).

[28] J. C. Montgomery, C. F. Baker, and A. G. Carton, Nature (London) 389, 960 (1997).

[29] M. Kulpa, J. Bak-Coleman, and S. Coombs, J. Exp. Biol. 218, 1603 (2015).

[30] J. Simmonds and D. N. MacLennan, Fisheries Acoustics: Theory and Practices (Wiley, New York, 2008).

[31] D. Helbing and P. Molnár, Phys. Rev. E 51, 4282 (1995).

[32] D. Helbing, I. Farkas, and T. Vicsek, Nature (London) 407, 487 (2000).

[33] H. I. Andersson and F. Jiang, Acta Mech. 230, 431 (2019).

[34] M. E. Porter, C. M. Roque, and J. H. Long Jr., Zoology 114, 348 (2011).

[35] D. J. Higham, SIAM Rev. 43, 525 (2001).

[36] P. Oteiza, I. Odstril, G. Lauder, R. Portugues, and F. Engert, Nature (London) 547, 445 (2017). 